\title{
Centrifugal force: an appreciation
}

\author{
Kern E. Kenyon \\ 4632 North Lane, Del Mar, USA; 'Corresponding Author: kernken@aol.com
}

Received 19 April, 2011; revised 2 May, 2011; accepted 8 May, 2011.

\begin{abstract}
The centrifugal force is used to increase the physical understanding of five examples taken from fluid dynamics, geophysics and the solar system, as well as four hypothetical orbital problems. Each example involves a balance of forces between the centrifugal force and one or two other forces, such as a pressure gradient and a component of the force of gravity. Among the examples chosen for examination are: the orbital motion of fluid particles in surface gravity waves, the boundary layer character of steady flow next to a curved rigid surface, the tornado, the rotating self-gravitating mass and the three-body problem.
\end{abstract}

Keywords: Centrifugal Force

\section{INTRODUCTION}

It may be easier for a trained dynamical oceanographer (the author) or meteorologist to take the centrifugal force seriously than for a practitioner of classical physics. In the field of physics there has existed for a long time a considerable amount of confusion surrounding the centrifugal force: is it real or fictitious? For example, see the descriptions of the centrifugal force in the various available dictionaries of physics, of which there are at least three. Here are two quotes from one such dictionary [1]. "Centrifugal force is said to be 'fictitious' force, and the use is best avoided." Also, "It is sometimes said that the centrifugal force is 'reaction' to the centripetal forcethis is not true." Both quotes illustrate the present state of confusion with respect to the centrifugal force. Throughout the discussions that follow support can be found for the second quote but none for the first one. Strangely, use of the centrifugal force in physics appears to have been restricted to purely circular motion. Why can't it be applied also to elliptical orbital motion? Exactly when the dissatisfaction with the centrifugal force began is not known; apparently no single event or person started it, unless it was Newton. He, on the one hand, pointed out that the flattening of the poles of the earth is direct evidence for the working of the centrifugal force, but, on the other hand, he used "centrifugal force" very sparingly in the Principia.

However, the physical oceanographer, operating in a relatively new field and not being hampered by all the physics history, learns in his first year of graduate work to balance the Coriolis force against a horizontal pressure gradient in order to help him understand the dynamics of the major permanent ocean currents. I believe the same thing happens when studying the large-scale wind systems in graduate courses in meteorology. Then it is only a small step to seek to balance the centrifugal force against another force, such as a pressure force, in order to increase understanding of some smaller scale motions. But there has never been much of a motivation to consider the centrifugal force in either ocean or atmospheric science. One reason for this is that the spatial scales of most weather features, even a hurricane, and ocean circulations are large enough to cause the Coriolis force to dominate over the centrifugal force.

In about 1982, a question was asked by me of myself regarding understanding the centrifugal force in surfaced gravity waves, since the fluid particles have an orbital motion, as indicated by observing small neutrally buoyant particles. This is discussed further in Section 2. By attempting to answer the question a chain of similar questions was triggered resulting in several totally different papers, all connected by a single thread (or 'most' common denominator): the centrifugal force. This chain of events was not planned or organized as such and the time sequence of the related publications did not always follow the order that is most convenient to put down here. But it all started with the surface gravity wave, a special subject of interest to me, before I found out from the dictionaries that I was not supposed to use the centrifugal force.

\section{SURFACE GRAVITY WAVES}

Thirty years ago, I began to think about the motion of the fluid particles in a surface gravity wave. Imagine the fluid particles in the fixed frame (fixed to the solid bot- 
tom of the body of water), which go round and round in circles while the sinusoidal wave shape propagates past the observer. During circular motion each fluid particle must experience a centrifugal force acting radially outward from the orbit's center. For the organized circular motion to be maintained and not break down into chaos or turbulence, there has to be an equal but opposite force to balance the centrifugal force. What is this other force? From looking into the literature it appears that nobody had ever asked this question before. The answer is a pressure force related to the different elevations of the sea surface and to the vertical fluid accelerations [2]. Immediately popping out of the force balance equation is the usual theoretical dispersion relation of these waves, derived on the basis of potential theory. There was a name already waiting in the oceanographic literature for the pressure/centrifugal force balance, the cyclostrophic balance, but it had never been applied to this example in the past.

At the time I asked the question of myself about how the centrifugal force on the orbiting fluid particles is balanced, my mind was not exactly prepared to do answer it. Ever since graduate school (in the 1960s), when I discovered a little known paper on water waves by Einstein [3], I had been under the spell of his elementary model that explained both the existence of the waves and their propagation speed with simple physics. He used a steady state model by assuming that the observer moves at the speed of the wave so that the wave shape remains steady and fluid under the wavy surfaces flows past the observer. This point of view had a pretty firm grip on my mind because of its usefulness for understanding some features of the waves. However, the observed orbital motion of the particles in the fixed frame does not come naturally out of the Einstein model. In other words, for all these years I had not been thinking much at all about the orbital movements of the particles. In another sense the question was ripening and just waiting to occur to me (or to somebody).

An opportunity came up, as it does every four years, to present a paper at an international meeting (IUGG), which happened at that time to be in Vienna. The timing was right to take into the public arena my thoughts on the centrifugal force as it applies to the surface gravity wave. My oral presentation (I also presented a poster) was scheduled for the largest lecture hall I had ever been in. Only a handful of people were there to hear the talk including my wife and our two kids. There I was up on a platform behind a podium far removed from my small audience in this giant room. One of the many amusing experiences of my academic career to think back on later.

After seeing this project through to publication, a re- lated question came up. According to the standard irrotational wave theory the particle orbits transform from circles to ellipses when the mean total depth of water becomes comparable to or less than a wave length and the long axes of the ellipses are horizontal, parallel to the mean free surface. The centers of rotation of the particles are the centers of the ellipses. So how does the cyclostrophic balance work in that case, since in general the centrifugal force cannot be anti-parallel to the central attractive (restoring) force? The restoring force, whatever its true nature is, must always point to the ellipse's center due to the inherent symmetry whereas the centrifugal force is always normal to the orbit. For guidance I turned to an undergraduate mechanics text I had used at MIT, which has a brief section on celestial mechanics. Of special interest to me then were the elliptical paths of the planets around the sun. I had gotten an " $\mathrm{A}$ " in this physics course many years before, but on revisiting the book I plunged into a really deep confusion, lasting for about two years. Sometimes in the book it seemed as if the author was dancing around the centrifugal force and other times not. For example, for purely circular motion the centrifugal force was occasionally mentioned whereas for elliptical orbital paths it was never used. A resolution of this confusion is described in Section 4.

In a way it was not completely logical for me look for help in astronomy for my problem with surface gravity waves in the ocean. Elliptical orbits are similar in both cases but there is a fundamental difference in the configurations with respect to the central attractive forces. For the planets, the gravitational force is centered at one focus of the ellipse whereas the restoring force center for the fluid particles in the surface gravity wave must be at the center of the ellipse, although the nature of the central attractive force was not yet identified at this point. At any rate, that is the path I chose to take, and I am now living with some of its consequences.

\section{TORNADOES}

Wouldn't a person think that among all observable natural geophysical phenomena the one with the fastest circular speed and relatively small radius of curvatureshould be a perfect subject for the application of the centrifugal force concept? However, there is little evidence of this in the literature on tornadoes (of course Newton never knew about tornadoes). Circular winds parallel to and near the ground have been measured to sometimes exceed $200 \mathrm{mph}$ by aiming Doppler radar beams at solid objects caught up in the tornado. What holds these orbiting winds together and keeps them from flying apart due to the outward acting centrifugal force? Evidently the core pressure must be a lot lower than the ambient pressure outside. Then how is such low pressure gene- 
rated?

Very few direct or indirect measurements have ever been made of the characteristics of a tornado, which is understandable in view of the high wind velocities and the dangers of getting too close to them. A man can stand against a $100 \mathrm{mph}$ wind, at a rather large angle from the vertical to be sure, but very few things, including meteorological instruments, can stand up to a $200 \mathrm{mph}$ wind. A lack of direct observations may have led to some rather mysterious ideas about tornadoes that have taken root in the minds of scientists.

Independent of any of my particular research topics that were occupying my time, my son took up the tornado as a project in grade school. He built a model tornado and used smoke to make the vortex motion visible. He got some assistance from a colleague of mine who knew some meteorology but he got little help from me (who didn't know any). Summers spent in Iowa, where his mother was born and raised and where his grandparents still lived, may have put this notion into his head. I could not help noticing from the sidelines, though, that his model tornado went from the ground up! After parts of many summers spent in Iowa, I have yet to see a tornado, except in still photographs and news videos. There is a strong suggestion in these visual aids, though, that the tornado comes down from the clouds to the ground. In other words, the vertical motion in the tornado is downward. For example, the funnel of the tornado is made visible because some cloud material is brought down to the ground with it. If one adopts this starting point, it makes the explanations of a few observed features easier, such as the funnel shape, the very large circular winds at the ground and the extremely low pressure inside.

Some key observations needed for a complete understandingof tornadoes are missing since they are difficult to obtain. Outside the tornado the air is always reported to be very warm and very humid, but is it cold and dry inside? Nobody knows for sure, except possibly one crazy storm chaser who apparently ran and dove into one and survived, according to a TV news report in May 2008. He came out saying that it was cool inside. Actually there is another storm chaser who probably knows the answer to some of these questions because at least once he successfully placed in the way of a tornado an instrument which had the capability of measuring temperature and humidity as well as pressure, and the instrument was not destroyed when the tornado went right over it on a paved road. However, so far only the record breaking low pressure has been reported to the public for some reason or other.

Suppose cool dry air, because it is heavier, falls down through the warm humid air, from the cloud to the ground. Then acceleration under gravity of the downward flow plus conservation of mass qualitatively accounts for the shape of the tornado's funnel, narrowing toward the ground. Suppose also that in or above the cloud base there has been generated somehow a horizontal circular motion which is comparatively weak. As it falls down this circulation is magnified by conservation of angular momentum as the funnel diameter becomes smaller. It is not difficult in this way to go from a relatively small horizontal velocity in the clouds to a 200 $\mathrm{mph}$ wind at the ground. What balances the outward centrifugal force is a pressure gradient in which there is low pressure at the core and relatively high pressure in the outside air. Low pressure is consistent with Bernoulli's law (pressure is least where the speed is greatest along a streamline) as the air falls (accelerates) downward at the center of the tornado. This is the gist of an elementary model [4] of a tornado in which the centrifugal force is strategically embedded.

\section{NEWTON'S HYPOTHETICAL ORBITS}

In addition to the one "real" Kepler problem, in the Principia Newton examined mathematically the following four hypothetical orbits of a single particle moving about a force center in two dimensions:

1) Elliptical orbit with the force center in the middle of the ellipse;

2) Circular orbit with the force center on the circumference;

3) Circular orbit with the force center in the middle of the circle;

4) Spiral with the force center at the pole of the spiral.

Some years ago five books were published within a few months of each other that all attempted to explain Newton's Principia to the educated public. Why this sudden and apparently widespread burst of interest in trying to understand the Principia, and why now after about 300 years? Earlier I had attempted on my own to read the Principia, but I couldn't see the forest for the trees, being lost in a tangle of theorems, corollaries, lemmas, etc. The reason for my searching the Principia was to hopefully relate my derivation of Kepler's laws to Newton's derivation of these laws, which is well-known to be the first theoretical prediction of the empirical laws. Perhaps I had found an independent derivation. But I could not follow Newton's geometrical and logical deductions, and I still can't. To this day I have trouble even locating the universal law of gravitation in the Principia!

However, one of the recent books allowed me to see better just what it was that Newton was trying to accomplish in one small part of the Principia. Now, although I still do not completely understand all the details of Newton's method, I saw that I can independently check his 
results on the four hypothetical orbits, plus his result of the Kepler problem, because my force balance method is the inverse of his method. He posed his orbital problems in the following way. Given the shape of the orbit and the location of the force center, find the functional form of the central attractive force that will keep a body in this orbit. My method is [5]: given the functional form of the central force and the force balance, find the shape of the orbit and its relation to the force center.

The Kepler problem is: for an elliptical orbit with the force center at one focus of the ellipse, find the form of the central attractive force. Newton obtained the inverse square law for the force in this case, i.e. the force varies as the inverse square of the distance between the focus and the body. My force balance method puts the inverse square law in and comes out with an elliptical orbit with the force center at one focus.

Four hypothetical orbital problems worked out in the Principia have not led to any practical applications so far, with one recent exception: the elliptical orbit with the force center at the center of the ellipse. Here Newton proved that the attractive force varies directly as the distance, like a linear spring, which is a special case of Hooke's Law. To the best of my knowledge Newton never used the term "spring" in connection with this hypothetical problem in the Principia, but it is known that Hooke and Newton were not the closest of colleagues. Recently a practical application of this orbital problem to surface gravity waves has been found, as discussed in Section 6.

By putting Newton's results for the central forces, one at a time, into my force balance equation in polar coordinates, that includes the centrifugal force, I can easily get the differential equations to be solved for the shapes of the orbits. In all cases the left side of the equation is the linear harmonic oscillator equation in polar coordinates where the variable of oscillation is the inverse of the radius (which may seem a bit unusual). Depending on the form of the force, the right side of the force balance equation can be linear or more generally nonlinear. Then if the corresponding shapes of the orbits that Newton began with are inserted into the differential equations, these equations can be solved and complete consistency is found between the two independent methods for all four hypothetical orbital problems in the Principia as well as for the Kepler problem. One striking offshoot is that analytic solutions in closed form, in terms of elementary functions, exist for two different differential equations that are both fully nonlinear, which one would never expect to find (one simply would not bother to search for it). Faced with such nonlinear differential equations the contemporary graduate student would automatically rush to the computer to solve them by means of numerical techniques.

This consistency between Newton's geometric method and my independent force balance method further strengthens the position that the centrifugal force is a real and useful force and not a fictitious one to be avoided. What is really needed, though, is a comparison between theory and measurements. That is not likely to happen with most of the hypothetical orbital problems. Perhaps the elliptical problem, with the force center at the center of the ellipse, may lead to such a comparison someday by means of a mechanical model (i.e. a mass rotating and vibrating on a spring, see Section 6).

Could one use the same force balance method with the centrifugal force to attack the famous unsolved 3-body problem of astronomy? This is a far more ambitious theoretical project than any that occurred to me before (or any that came after either).

\section{THREE-BODY PROBLEM}

At the end of the 1990s, I attempted to solve the celebrated 3-body problem in a plane (i.e. in two dimensions) using my force balance method, including the centrifugal force, and I got farther than I had expected to get on the first try. However, the equations started to look a little messy so I stopped. But the recent progress with Newton's Principia, in which all five of the orbital problems were found to be consistent with the force balance method, gave me the extra courage to try again. On a vacation trip to New Mexico I did not have any notes with me, but nevertheless I sat down and drew a set of traingles connecting the three masses. In doing so I just happened to define two angles differently than before, and this produced enough of a simplicity in the trigonometry that it eventually led to an initial breakthrough, which allowed me to proceed further than I had done previously. Without my books and papers I had to rederive most of the necessary mathematical relations; a few I remembered but had to check. Four pages in the notebook were enough to contain the derivation of the kernel of the solution of the 3-and 4-body problems, and then the generalization to the n-body problem was just a relatively simple extension of the same procedure.

As it turns out now my original solution had errors in it, which were pointed out by one peer reviewer in due course, but these mistakes were corrected one by one until the paper was finally accepted after about a twoyear review period. Of course I am very grateful to this particular reviewer, whoever he or she is. The word "solution" is not quite accurate, since what I have done so far is to set up the differential equations to be solved for the orbits of the gravitating masses. I have not actually solved these equations and I am not the most likely person to do so either. The equations are strongly nonlinear 
and in the general case almost certainly must be addressed by numerical methods, about which I am almost totally ignorant. Only after that work is done can a comparison between theory and measurement be carried out, upon which the theory will either stand or fall.

For 300 years the 3-body problem has teased researchers, mathematicians, physicists, and astronomers. It gave Newton a headache. It gave Euler a headache. Many mathematicians and physicists devoted a considerable amount of time to the problem. A 1967 Encyclopedia of Science estimates that about 1000 papers had been published on the 3-body problem. There must be considerably more papers than that now, and there may even have been more then.

On the other hand, I never declared in the beginning that I was working on the 3-body problem, but rather bumped into it sideways: picked it up for a day, put it down for three years, and then picked it up again for a few days and thought I had finished with it (until the reviewer got hold of it). If I have really solved this famous problem, which at the moment I think I have, it will be something to wonder at briefly before hopefully going on to write other papers. Then I predict that the physicists will more readily accept the centrifugal force as being a real useful tool.

The actual working out of the setting up of the solution of the 3-body problem took a few interesting twists and turns through the review process. One anonymous revie- wer thought $\mathrm{I}$ had made a fundamental mistake in setting up the force balance equations for the three masses in the first place. I was so sure of myself that I did not come to realize that he was right for a considerable time, but kept trying to justify my position. However, the reviewer was unyielding. Eventually, with the help of some friends on a particular point of the problem (and mostly by e-mail), I saw my mistake and stayed up all night correcting it.

Then the same reviewer had another major objection to my suggested method of solution of the corrected force balance equations. Again I was sure of my proposed method, because it was a straight forward extension, I thought, of an earlier published paper of mine on the 2-body or Kepler problem. I was not happy with the result either and could not think of a way around the obstacle. None of my friends came back with any useful information when I asked them about it. Finally, the reviewer pushed me hard enough that I found a way to solve the difficulty which was at the same time more mathematically correct and not physically inconsistent, but it confused me because of my published results and made me think I had made a mistake before. There was a tug of war going on between my feelings of happiness for having found a way to solve the 3-body problem and my confusion over the way I had previously solved the 2-body problem. Gradually the feeling of happiness began to win out after a few anxious days. Naturally I am indebted to this reviewer for his patience and persistence in sticking to his position, and the editor was unbelievably great throughout; a real test for all three of us actually.

But this was not the end of the story either, because I had gone out on a limb in offering an opinion about a way that the equations could be solved numerically. One more time the reviewer was very critical of my suggestion, and probably rightly so. After taking this section out of the paper, the editor finally accepted it for publication [6]. No solutions are contained in the paper, and no comparisons are made between theory and measurement. These important pieces of work are left for the future. About one year and a half after the review process was finished the paper finally saw the light of day.

\section{ROTATING MASS ON A SPRING}

Since Newton's day one of his hypothetical orbital problems, an elliptical path with the force center in the middle of the ellipse, has come to be associated with a practical situation: particle orbits of the surface gravity wave [8]. In this case Newton discovered that the central force needed to keep a mass in its orbit varied directly as the distance the body was from the force center. In other words the force behaved like a linear spring. Thus the present exploration of problems incorporating the centrifugal force has come full circle, so to speak, starting and ending with the surface gravity wave. However, the end of the tale has not yet been reached because two more sections follow this one.

Another way this particular hypothetical problem could become a practical one is by simply attaching a mass to a spring, anchoring the other end of the spring, giving the mass a push while restricting the motion to the horizontal plane, for example. But there are two idealizations of the theoretical spring that may take a bit of creative engineering in order to build such a device: the spring is supposed to be mass less and have zero equilibrium length. Also friction must be eliminated (or greatly reduced).

A noteworthy property of such a theoretical device, apparently not noticed by Newton, is that the orbital period of the mass is independent of the size, shape and orientation of the elliptical orbit. Such a machine would be a very good clock in the sense that in spite of several types of disturbances of the mass, within its plane of motion, it will keep perfect time!

The period of the mass rotating on a spring is directly related to the spring constant and to the mass of the rotating body but totally independent of the major and minor axes of the elliptical trajectory as well as its orienta- 
tion. This is in stark contrast to Kepler's third law by which the elliptical orbital period of a planet around the sun does depend on the major axis of the planet. [It seems a bit strange, when one thinks about it, that of the two length-scales of the elliptical orbit, the period only depends on one of them (the major axis).]

Identifying the surface gravity wave with Newton's hypothetical orbital problem of an elliptical orbit due to a force emanating at the center of the orbit did not come easily or by a straight path although it very well could have. Circles are special cases of ellipses and the fluid particles in propagating surface gravity waves move in circles for infinitesimal amplitudes in deep water. Also in the cyclostrophic balance of forces on each particle the outward centrifugal force is equal and opposite to the pressure force, and each of these two forces varies linearly with the distance of the particle from the center of its orbit, the attractive pressure force acting like a spring.

From another point of view, the radii of the circular particle orbits are known observationally to decrease with increasing mean depth. Therefore, all radii from zero to the wave amplitude exist simultaneously in the water column. And each particle makes one complete revolution around its orbit in the same length of time, the wave period. In other words, the orbital period of the particle is independent of the size of the orbit! There is only one known orbital problem with this characteristic: the mass rotating in a plane on a linear spring.

When the mean depth is comparable to or less than the wavelength the particles orbit in ellipses, theoretically, with the major axes being parallel to the flat bottom. Both major and minor axes decrease with increasing depth but the period of each orbit remains constant, the wave period. This example comes closer to being an analogue of Newton's hypothetical orbital problem.

\section{ROTATING SELF-GRAVITATING MASS}

An extension of the simple model of the mass rotating and vibrating on a spring in two dimensions is as follows: a rotating self-gravitating mass in three dimensions, or a model of a planet made of either solid or gaseous material. Here, although counter-intuitive for a solid, compressibility is the key characteristic for solving the problem. Obviously each particle completes its orbit in the same period of time, the rotational period of the planet, independent of the size of the orbit. This extension might seem a bit farfetched until it is realized what the connecting link is. At an interior point of a spherical mass of uniform density the gravitational attraction acting on it is known to be a linear function of the distance of the point from the center of the body. In other words, the attrac- tion inside the body acts like an ideal linear spring, which is both mass less and has zero equilibrium length. The linear spring behavior is the common feature, along with the centrifugal force, between the two- and threedimensional problems.

One difference between the two and three dimensional problems is that for the rotating self-gravitating body there are three instead of two forces that need to be balanced. In addition to the restoring spring force due to gravitational attraction and the outward centrifugal force there is also a pressure force inside the body. It needs to be demonstrated that the pressure force also depends linearly on the distance from the center of the mass. Then all three forces are directly proportional to the radius, since the centrifugal and gravitational forces already are, and when they are balanced, the radius drops out of the problem. The result is a formula for the rotational frequency of the body that is independent of the radius, and also independent of the latitude, as it turns out [8].

If the mean density and the rotational periods of the six planets that have nonzero values of their flattenings are supplied from measurements, the model accounts for $35 \%-75 \%$ of the observed flattenings. Increasing density with decreasing radius inside the planets accounts qualitatively for the discrepancies. Therefore, what appears to be a rather rough kind of agreement between data and a theory involving the centrifugal force has been obtained. Hopefully in the future a closer correspondence can be established.

The balance of forces leads both to the equilibrium form of the rotating body as well as the stability of certain perturbations of it. For the equilibrium form the small excess of the equatorial radius over the polar radius, due to the stretching of the compressible solid material under the centrifugal force, trails off like the square of the cosine of the latitude. Therefore the equilibrium form is not strictly an ellipsoid. Superimposed on this equilibrium form is a perturbation that is taken to be in planes perpendicular to the rotation axis of the body. Such a perturbation might be caused, for example, by the gravitational attraction of a sufficiently distant object, like a moon or a sun. Before the perturbation the planes normal to the rotation axis are circles, but after the perturbation they are more than likely concentric ellipses with centers on the axis of rotation. Thus there are two bulges on opposite sides of the mass. Consequently, in one rotational period there are two high and two low bodily tides. The model in its present form has nothing to say about the expected amplitude or phase of the bulges in relation to the given perturbing force. In any case the seeds have been planted for a possible alternative explanation for why two tides should occur per 
day, at least in the solid earth.

\section{FLOWS ADJACENT TO A CURVED SOLID BOUNDARY}

A relatively new type of boundary layer, called the curvature boundary layer [9], that involves the centrifugal force but no friction, comes last in this list of topics that was initiated by wondering about the orbital motion of fluid particles in a surface gravity wave. Consider fluid flowing steadily next to a smooth flat rigid boundary with one bump protruding into the flow. Observations will show that if the fluid speed is not too great and the slopes of the bump are small, then the fluid will follow the bump's contours without separation or eddies being shed off the back face. Also flow visualization techniques will illustrate that the perturbed velocity caused by the bump only penetrates a finite distance above the bump. Within a few lengths of the bump, marked off in the vertical direction, the curved streamlines have degenerated into straight lines. This is an inviscid boundary layer due to the working of the centrifugal force on the curved flow. When measurements indicate that flow near a curved solid boundary is steady, there must be an equal but opposite force to counter balance the centrifugal force wherever it exists. This force can only be a pressure gradient under the given circumstances.

Bernoulli's law states that where the speed is greatest the pressure is least along a streamline in steady frictionless flow. For the streamline just going over the top of the bump the pressure is least at the top of the bump because the speed is largest there by conservation of mass. Through each cross-section equal amounts of mass must flow in equal times. Above the top of the bump the cross-section is least due to the fact that the perturbed velocity dies off upward away from the bump. Conservation of mass only works in this way if the complete trailing off of the perturbed velocity takes place within a finite distance of the bump. Assuming incompressibility of the fluid is fine but not essential.

To see why it is that the disturbed pressure also decays upward over the bump, look at the force balance in the cross-stream direction: the pressure gradient equals the centrifugal force. By convention the radius of curvature of the flow over the bump is positive and the square of the flow speed is positive so the centrifugal force is positive. That means the counterbalancing pressure gradient must be positive. In other words the pressure, which has a deficit at the top of the bump, increases upward and will equal the ambient pressure some finite distance away from the bump.

One of the consequences of the "vertical" gradients in pressure, normal to the bump, is that there are "horizontal" variations in pressure parallel to the bump. Since the bump is assumed rigid, these pressure variations will not distort its shape. Relatively low pressure at the bump is the origin of the well-known lift force on the bump

As the pressure increases upward above the bump the speed of the horizontal flow must decrease in accordance with Bernoilli's law. For a smaller flow speed the upward centrifugal force is smaller requiring a smaller pressure gradient to balance it. So not only does the pressure increase upward but the pressure gradient decreases upward. Therefore, pressure and velocity perturbations, caused by the presence of the bump, are confined to the neighborhood of the bump in a boundary layer configuration. For flow past a cylinder it turns out that the pressure perturbation decays at a faster rate than the velocity perturbation does over the top (and bottom) of the cylinder. This appears to be a new result in theoretical fluid dynamics [10].

\section{REFERENCES}

[1] Daintith, J. (1998) The facts on file dictionary of physics. Facts on File, New York.

[2] Kenyon, K.E. (1991) Cyclostrophic balance in surface gravity waves. Journal of the Oceanographic Society of Japan, 47, 45-48. doi:10.1007/BF02301754

[3] Einstein, A. (1916) Elementare theorie der wasserwellen und des fluges. Naturwissenschaften, 4, 509-510. doi:10.1007/BF01491471

[4] Kenyon, K.E. and Kenyon, D.C. (1989) An elementary model of a tornado. Geophysical Research Letters, 16, 1281-1283. doi:10.1029/GL016i011p01281

[5] Kenyon, K.E. (1999) Newton's hypothetical orbits independently derived. Physics Essays, 12, 39-43. doi:10.4006/1.3025371

[6] Kenyon, K.E. (1999) The n-body problem solved in two dimensions. Physics Essays, 12, 197-203. doi:10.4006/1.3025377

[7] Kenyon, K.E. (2001) Exciting a rotation mass on a spring without change to its rotation rate. European Journal of Physics, 22, 471-475. doi:10.1088/0143-0807/22/5/301

[8] Kenyon, K.E. (2002) Equilibrium and perturbed forms of a rotating self-gravitating mass. Physics Essays, 15, 290-296. doi: $10.4006 / 1.3025530$

[9] Kenyon, K.E. (2003) Curvature boundary layer. Physics Essays, 16, 74-85. doi:10.4006/1.3025569

[10] Kenyon, K.E. (2011) Flow past a cylinder (submitted for publication). 